\title{
Nanocrystal based orally disintegrating tablets as a tool to improve dissolution rate of Vortioxetine
}

\author{
Original \\ Article \\ Eknath Ahire, Shreya Thakkar, Yogeshwari Borade, Manju Misra \\ National Institute of Pharmaceutical Education and Research (NIPER), Ahmedabad, \\ Gujarat-380054, India.
}

\begin{abstract}
Major depressive disorder (MDD) or depression is a heterogeneous mental illness which have direct effect on patient's behavior and cognition; causing constant feeling of unhappiness and loss of concern in earlier enjoyed events. Vortioxetine (VOR) is a new atypical serotonergic antidepressant reported for the treatment of major MDD and has its use in various psychological disorders. Despite its therapeutic potential, it has poor water solubility which creates problem in delivery. Nanocrystal based orally disintegrating tablets (ODTs) are proposed to enhance water solubility and dissolution rate of VOR. Nanosuspension was prepared by nanoprecipitation method after optimizing various parameters. The formulated nanosuspension was effectively converted to solid powder by the lyophilization technique with emphasis on parameters like nature/concentration of cryoprotectants. Prepared nanocrystals were characterized for solid state of drug present, morphology and size after re-dispersion. The powders were then subsequently compressed into ODTs and dissolution profiles of the tablets were compared. The dissolution profile of the ODTs prepared from nanocrystals showed superior dissolution profile in comparison to ODTs prepared from untreated drug.
\end{abstract}

Key Words: Lyophilization, Nanocrystals, ODTs, powders, solubility enhancement.

Received: 10 February 2020, Accepted: 26 April 2020

Corresponding Author: Manju Misra, Associate professor, Graduate school of Pharmacy,Gujarat Technological University Gandhinagar Campus Nr. Government Polytechnic K-6 Circle, E-4 Electronic Estate G.I.D.C, Sector - 26, Gandhinagar-382028- Gujarat. Tel.:: 9099069333, E-mail: mmisraniper@yahoo.com

ISSN: 1110-0930, 2020 Vol.58, No. $1 \& 2$

\section{INTRODUCTION}

Major depressive disorder (MDD) or depression is a heterogeneous mental illness which have direct effect on patient's behavior and cognition; causing constant feeling of unhappiness and loss of concern in earlier enjoyed events ${ }^{[1]}$. Depression recurrently goes unrecognized and untreated; and may be a causative factor of suicidal attempts and impaired interpersonal relationship in society as well as at work space. People may experience it once in life or as multiple episodes in lifetime ${ }^{[2]}$. Depression is one of the very common psychological disorder and as per the reports of World Health Organization (WHO), worldwide 350 million people suffer from depression, that is closer to about $5 \%$ of the world's population ${ }^{[3]}$. It is one of the leading cause of disability and its prevalence is more in women as compared to men ${ }^{[4]}$. The treatment of MDD includes different categories of antidepressants such as selective norepinephrine reuptake inhibitors (SNRI), selective serotonin reuptake inhibitors (SSRI), tricyclic antidepressants, atypical antidepressants, etc. ${ }^{[5]}$.

Vortioxetine (VOR) is a new atypical serotonergic antidepressant reported for the treatment of MDD and has its use in various other psychological disorders. VOR works as antagonist of 5-HT3, 5-HT1D, 5-HT7 and has agonist and partial agonist activities for 5-HT1A and
5HT1B respectively ${ }^{[6]}$. It acts as a serotonin reuptake inhibitor and also has serotonin receptor modulating activity. Mode of action for VOR is not fully understood but it is hypothesized that VOR binds with a greater affinity to the serotonin transporter and its antidepressant actions are considered to be secondary; due to the increase in serotonin levels in the brain as a result of reuptake inhibition ${ }^{[7]}$. Despite its potential in the treatment of MDD, main hurdle in delivery of this drug candidate is its poor water solubility. Vortioxetine was approved in 2013 by Food and Drug Administration for the MDD management under the brand name of Brintellix having drug as a hydrobromide salt ${ }^{[8]}$. Due to the basic nature of drug as well as comprising of secondary amine functional group ( $8.85 \mathrm{pKa}$ value of $\mathrm{N} 1$ ), various salt forms can easily be made with acids including fumarate, $\mathrm{HCl}$ and many more. However these salt forms are very prone for conversion to different polymorphic forms which again have possibilities to change physicochemical properties of developed salt ${ }^{[9]}$. Thus there is a basic need to work upon some other solubility enhancement techniques for this drug candidate.

Physical/chemical modification and formulation based approaches are also in use since long ago for solubility enhancement, amongst which size reduction is one of the basic concept, having prime importance in the pharmaceutical field. Reduced particle size helps in 
improving bioavailability of compounds by overcoming erratic drug absorption ${ }^{[10]}$. Nanoparticles are known to improve performance of drug candidates in a variety of dosage forms. Modified performance of this delivery system include solubility enhancement, delivery to specific site and many more ${ }^{[11]}$. Nanocrystal is one amongst the best known techniques for solubility enhancement which also offers the various advantages over the other solubility enhancement techniques.

Nanocrystals are particles of pure drug in nano size range stabilized with the aid of little amount of stabilizer. These differ from other nanomaterials in a way that these are composed of active pharmaceutical ingredient contrary to serving as drug carriers by virtue of excipients that is usually the case with micelles, liposomes and polymeric nanoparticles ${ }^{[12]}$. Some authors have expanded this definition of nanocrystals to include amorphous form of drug as well, and accept both crystalline and amorphous form in its definition ${ }^{[13,14]}$. Drug particles are simply covered with layer of surfactant or polymer which acts as a stabilizer and omits the requirement of any other excipients. Because of its size in nanometer range, nanosuspension offers a high surface area to volume ratio, improving saturation solubility as well as rate of dissolution ${ }^{[15]}$.

Owing to the advantages offered by nanocrystal, it is proposed to formulate orally disintegrating tablets (ODTs) of VOR to enhance its water solubility as well as the rate of dissolution ${ }^{[16]}$. Nanosuspension of VOR was prepared by nanoprecipitation method after optimizing various parameters. The prepared nanosuspension was effectively converted into solid powder with specific focus on parameters affecting final product like nature and concentration of cryoprotectants. Nanocrystalline dispersion was characterized for state of drug present, morphology and size after redispersion. Subsequently the lyophilized powders obtained were compressed to ODTs and the rate of dissolution were compared.

\section{Experimental section}

\subsection{Materials}

VOR was received as a gift sample from Amneal pharmaceuticals Pvt. Ltd. (India). Pluronic ${ }^{\circledR}$ F-127, Sodium lauryl sulphate (SLS), Trehalose dihydrate (TD), Hypromellose (HPMC) and Pluronic $\AA$ F-68, were procured from Sigma-Aldrich (Germany). Maltose monohydrate (MM) and Sorbitol were supplied by HiMedia Laboratories (Mumbai, India). Disodium hydrogen phosphate, sodium chloride and potassium dihydrogen phosphate were procured from Fischer scientific (USA). Mannitol was procured from Spectrochem Pvt. Ltd. (Mumbai India). Water used in all experiments, was obtained from a Milli ${ }^{\circledR}-\mathrm{Q}$ Biocel, Millipore ${ }^{\circledR}$ (USA) assembly. All other solvents and reagents used in formulation were of analytical grade and used as such without further purification.

\subsection{Methods}

\subsubsection{Drug-excipient compatibility studies}

The drug- excipient compatibility studies were done by means of differential scanning calorimetry (DSC ${ }^{[17]}$. Binary ratios of VOR-excipients were prepared and were checked for any kind of interactions using an Indium calibrated auto DSC basic unit (NETZSCH) with provision of a refrigerated cooling system was used for the measurements during the study. Approximately 1.5- $4.0 \mathrm{mg}$ of different binary mixtures were weighed in an aluminum pan and was allowed to heat from $0{ }^{\circ} \mathrm{C}$ to $250{ }^{\circ} \mathrm{C}$, using nitrogen as an inert gas. In this study, the standard (reference) used was empty DSC pan.

Binary ratios (1:1) of VOR -excipients were prepared and were checked for any kind of chemical interaction using Fourier-transform infrared spectroscopy (FTIR) spectroscopy. All the measurements were done using Alpha Opus 7.5 instrument (Bruker, USA). The mixtures were examined following formation of $\mathrm{KBr}$ pellet. In this method, the pellets were formed by compressing about $2 \mathrm{mg}$ of mixture triturated along with moisture free $\mathrm{KBr}$ using hydraulic press. These were then analyzed in the range of 4000-400 $\mathrm{cm}^{-1}$.

\subsubsection{Preparation of nanosuspension}

Conventional nanoprecipitation method was tried as preparation method for VOR nanosuspension. The nanoprecipitation of VOR was achieved by the solventantisolvent technique. The solvents were selected from dichloromethane (DCM), methanol, ethanol and acetone. Different surfactants screened as stabilizer were Pluronic ${ }^{\mathbb{R}}$ F-68, Pluronic ${ }^{\circledR}$ F-127, HPMC, TPGS and SLS alone as well as in combination with each other. Various material attributes and processing conditions viz. selection of solvent, stabilizer type, drug to surfactant ratio, solvent to anti-solvent ratio and stirring speed were optimized to get preferred particle size and $\mathrm{PDI}^{[18]}$. One variable at a time approach was used in order to optimize the formulation conditions. Table 2 summarizes all the attempts made to get acceptable size and PDI (polydispersity index). All the batches were formulated in triplicates $(n=3)$.

\subsubsection{Solidification of nanosuspension}

Different types of techniques are used for solidification in order to avoid the physical and chemical instability issues related to drug. Solidification leads to hindered particle motion and reduced chances of collision which ultimately results in physical stability of drug. Also, the elimination of water during solidification significantly decreases the most of the chemical reactions responsible for chemical instability of drug. Furthermore, it is well known that the chemical and physical interactions occurs rapidly in liquid state in comparison to solid state, and thus solidification results in stabilization of the system. Various solidification techniques like freeze drying, hydrogel formation, spray drying, palletization and electrospinning 
had been explored by the researchers that are suitable to convert nanosuspension into nanocrystals ${ }^{[19]}$. From all of these techniques freeze drying and spray drying are the ones which have been explored much more by scientists working in this area ${ }^{[19,20]}$. Nanosuspension of the optimized batch was lyophilized at constant temperature and pressure of $-80^{\circ} \mathrm{C}$ and $5 \mathrm{mT}$ (ilshinBioBase, South Korea) respectively. Mannitol, maltose, TD, and D-sorbitol were screened for their use as cryoprotectant ${ }^{[19]}$. Concentrations of $1 \%, 2 \%, 5 \%$, and $10 \%$ of aforementioned cryoprotectants were screened utilizing freeze-thaw studies to optimize the one with better effect. Nanosuspension with/without selected cryoprotectants was subjected to freeze and thaw cycle at $-80^{\circ} \mathrm{C}$ for $72 \mathrm{~h}$ at room temperature respectively. After thawing, particle size, PDI and zeta potential of the formulation was determined. The cryoprotectants providing $\mathrm{Sf} / \mathrm{Si}$ ratio (where, $\mathrm{Sf}$ is the final particle size after thawing, and $\mathrm{Si}$ is initial particle size before freezing) less than 1.3 were selected for further optimization ${ }^{[21]}$. Finally samples were lyophilized following freezing at $-80{ }^{\circ} \mathrm{C}^{[22,23]}$.

\subsubsection{Formulation characterization}

\subsubsection{Analysis of particle size, PDI and zeta potential}

A known quantity of lyophilized nanocrystals was dissolved in $5 \mathrm{ml}$ distilled water (Milli ${ }^{\circledR}-\mathrm{Q}$ water) and shaken properly so as to get a uniform dispersion. This was then analyzed using Zeta sizer which is based on the principle of dynamic light scattering (Nano ZS series, Malvern) for size and PDI. Zeta potential was measured with the help of same instrument. All the measurements were done in triplicate $(n=3)$.

\subsubsection{Particle morphology and surface roughness analysis}

The surface morphology analysis of lyophilized formulation was carried out using Scanning electron microscope (SEM). Study was conducted for analysis of particle surface morphology of pure drug, physical mixture and lyophilized nanocrystals. The samples were prepared by mounting the powder onto a metal stab with the help of a double sided conductive adhesive tape and platinum coated sputter in an inert gas atmosphere and then the SEM analysis (JSM-7600F, Jeol) were carried out at an excitation voltage of $3 \mathrm{kV}$.

To determine the changes in surface roughness of nanocrystals in comparison to the untreated drug, Atomic Force Microscopy (Multimode 8, Bruker, USA) was done. Scan speed was set at $0.5 \mathrm{~Hz}$ and scan sizes were taken from $100 \mathrm{~nm}$ to $800 \mathrm{~nm}$. Before analysis, sample was prepared by dispersing sufficient amount of sample in water and glass slides were prepared by drop casting method. The data was represented in topographic and phase imaging mode. After drop casting, the sample was subsequently dried at ambient conditions in order to prevent movement of particles.

\subsubsection{Characterization of physical/chemical state of drug present}

$\mathrm{X}$-ray diffraction (XRD) and DSC were carried out to verify the state of drug present (amorphous or crystalline) in final lyophilized nanocrystals. The X-ray powder diffractograms were recorded at room temperature by a diffractometer (GNR APD 2000 Pro) using a Modern SAX software. The sample was spread evenly on a holder and scanned for $2 \theta$ range of $3-40^{\circ}$ with a step size of 0.01 and at a scan speed of $0.1 \mathrm{sec}$. DSC and FTIR analysis was performed with conditions mentioned in section 2.2.1.

\subsubsection{Drug assay}

Analytical method for drug was validated using high performance liquid chromatography (HPLC) Agilent system. C18 Kromasil column $(250 \mathrm{~mm}$ x $4.6 \mathrm{~mm}, 5 \mu)$ was utilized with mobile phase of methanol: ammonium acetate buffer $(\mathrm{pH} 4)$ in ratio 75:25. Analysis was done at $230 \mathrm{~nm}$ with flow rate of $1 \mathrm{ml} / \mathrm{min}$ and injection volume of $5 \mu \mathrm{L}$. Accurately weighed lyophilized sample $(5 \mathrm{mg})$ was solubilized in $1 \mathrm{ml}$ of Milli ${ }^{\circledR}-\mathrm{Q}$ by subjecting to vortexing and sample was then analyzed (after possible dilution required) for drug assay by validated analytical method. The dissolved sample was filtered with $0.2 \mu \mathrm{m}$ filter before analysis.

\subsubsection{Formulation of Orally Disintegrating Tablets (ODTs)}

\subsubsection{Evaluation of flow and compressibility index}

Bulk density, tapped density as well as angle of repose were determined to assess flow properties and compressibility behavior of final blend prepared. Bulk and tapped density were determined by the method as previously described ${ }^{[24]}$. The following equations were used to calculate the compressibility index and Hausner's ratio (where $\rho$ Tapped is tapped density and $\rho$ Bulk corresponds to bulk density).

$$
\begin{aligned}
\text { Compressibility index } & =\frac{\rho_{\text {Tapped }}-\rho_{\text {Bulk }}}{\rho_{\text {Tapped }}} \times 100 \\
\text { Hausner's ratio } & =\frac{\rho_{\text {Tapped }}}{\rho_{\text {Bulk }}}
\end{aligned}
$$

Angle of repose which is the measure of flow property of powder is an angle formed by the edge of pile with the horizontal base after falling of sample powder from a funnel which is fixed at particular height from horizontal base. Height of the cone formed (h) and radius of the base (r) were measured and angle of repose was calculated by the following equation.

$$
\theta=\tan ^{-1}\left(\frac{h}{r}\right)
$$




\subsubsection{Preparation of ODTs}

Tablet blend of lyophilized nanocrystals as well as untreated drug was prepared along with excipients for the preparation of ODT (Table 1) by direct compression technique. All the excipients were sieved from a coarse sieve of 40 mesh size. After geometric mixing of drug dose with all the excipients, powder blend was mixed with talc and magnesium stearate as lubricants for 5 mins. Finally, the dry mixture was compressed by means of $6.00 \mathrm{~mm}$ flat punches on 8 station rotary tablet compression machine (Kambert, Gujarat, India).

Table 1: Composition of ODT

\begin{tabular}{ll}
\hline \multicolumn{1}{c}{ Excipient } & \multicolumn{1}{c}{ Weight Per Tablet } \\
\hline VOR / Lyophilized & $\begin{array}{l}5 \mathrm{mg} \text { or equivalent to } 5 \mathrm{mg} \text { of } \\
\text { drug } \\
\text { nanocrystals }\end{array}$ \\
Mannitol & $10 \mathrm{mg}$ \\
Pharmaburst 500 & $85 \mathrm{mg}$ \\
Talc & $1.5 \%$ \\
Mg Stearate & $1.5 \%$ \\
\hline
\end{tabular}

\subsubsection{Evaluation of ODTs}

Evaluation of prepared ODTs for hardness (by Monsanto hardness tester), weight variation, friability, and dissolution profile and disintegration time was performed. Briefly, three tablets from each of the batch were randomly selected and crushing strength required was determined by hardness tester in $\mathrm{kg} / \mathrm{cm}^{2}{ }^{[25-28]}$.

Wetting time and water absorption ratio of the prepared tablets were also determined that indicate the ease of disintegration of tablet in the buccal cavity. Briefly, a 8.5 $\mathrm{cm}$ piece of folded (twice) tissue paper was placed in a petri dish ( $8.5 \mathrm{~cm}$ internal diameter) containing about $8 \mathrm{~mL}$ of aqueous eosin dye solution $(0.5 \%)$. Each tablet $(n=6)$ was then placed on the tissue paper carefully and the time required for the dye solution to appear on the upper surface of tablet indicating complete wetting was recorded as the wetting time. After that, the wetted tablet was weighed and the water absorption ratio (R) was calculated using following equation.

$$
R=\frac{W_{t}-W_{o}}{W_{o}} \times 100
$$

Where, $\mathrm{W}_{\mathrm{t}}$ is the tablet weight after complete wetting and $\mathrm{W}_{\mathrm{o}}$ is the initial weight of the tablet ${ }^{[29-31]}$.

Disintegration time was determined by a tailored disintegration method $(n=6)^{[32]}$. Briefly, about $10 \mathrm{ml}$ of water was taken in petri dish and tablet was cautiously placed in the center of the Petri dish. The time required for the tablet to wholly disintegrate was noted by means of a stop watch.
Weight variation was studied by taking 20 tablets (selected on random basis) and calculating average weight followed by comparison of weights from individual tablets with average weight.

Friability testing was done utilizing Roche friabilator. Pre-weighed tablets were taken and subjected to 100 revolutions in the friabilator, dropping the tablets from 6 inches in each revolution. Friability of tablets was determined by the equation mentioned below.

$$
\text { Friability }=\frac{W_{1}-W_{2}}{W_{1}}
$$

Where, $\mathrm{W}_{1}$ is initial weight and $\mathrm{W}_{2}$ final weight.

In vitro dissolution study for ODTs formulated using lyophilized nanocrystals and untreated drug was performed in $500 \mathrm{ml}$ of dissolution medium (SSF and $0.1 \mathrm{~N} \mathrm{HCl}$ ). The temperature of the medium during the experiments was kept $37 \pm 0.5^{\circ} \mathrm{C}$ and rotational speed of $50 \mathrm{rpm}$. The sample $(5 \mathrm{ml})$ were withdrawn at the time intervals of 5 , $10,15,20,25,30,40$, and 60 minutes and at each time point the fresh dissolution medium was used to replace withdrawn sample. Withdrawn samples were subjected for analysis a using HPLC method as described in preceding section.

\section{RESULTS AND DISCUSSION}

\subsection{Drug-excipient compatibility studies}

HPMC, SLS, Pluronic ${ }^{\circledR}$ F-127, Pluronic ${ }^{\circledR}$ F-68 and TPGS were taken for screening of physically compatible stabilizer. DSC curves for drug revealed three endothermic events at $100{ }^{\circ} \mathrm{C}, 228{ }^{\circ} \mathrm{C}$ and $233{ }^{\circ} \mathrm{C}$ corresponding to the peak of moisture and two polymorphic forms of drug. Binary mixtures prepared with HPMC, Pluronic ${ }^{\circledR}$ F-68 and Pluronic ${ }^{\circledR}$ F-127 showed retention of drug peak for single polymorph along with characteristic peaks of their own (Figure 1). In case of SLS and TPGS mixtures drug peak was found to be absent. For further clarification, hot stage microscopy (HSM) was performed (Figure 2) and events visualized suggested that the solubilization of drug particles in melt of both these stabilizers which clarifies the reason for absence of endothermic event in these cases.

Results obtained for FTIR analysis showed retention of all the peaks for drug (at $3284 \mathrm{~cm}-1,2929 \mathrm{~cm}-1$, $1473 \mathrm{~cm}-1,1229 \mathrm{~cm}-1,762 \mathrm{~cm}-1$ corresponding to N-H stretching, C-H stretching, $\mathrm{C}=\mathrm{C}$ stretching, $\mathrm{C}-\mathrm{H}$ stretching, $\mathrm{C}-\mathrm{S}$ stretching respectively) in all of the prepared binary mixtures of the drug with excipients (Figure 3). Peak broadening was observed for some binary mixtures which could be due to the H-bonding interactions ${ }^{[33]}$. Hence all the selected excipients were found to be both physically as well as chemically compatible with the drug and thus were taken further for screening of stabilizer by preparing preliminary batches of nanosupension. 


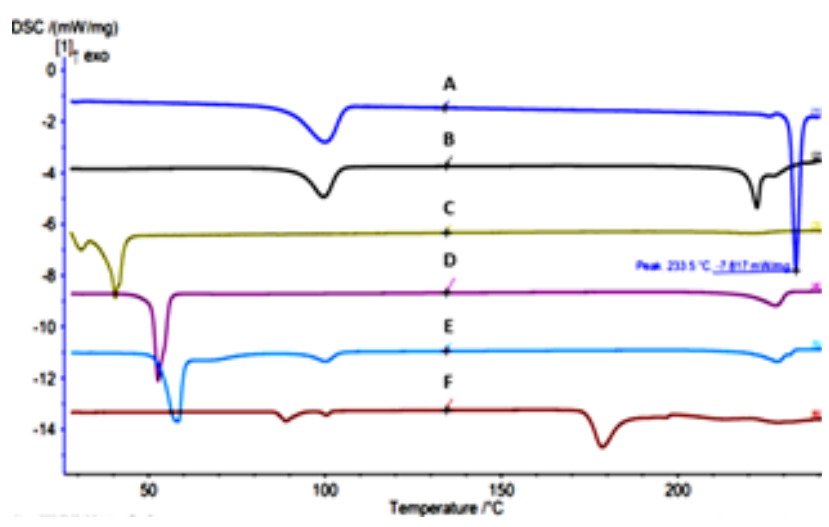

Fig. 1: DSC thermograms of A: Drug, B: Drug + HPMC, C: Drug + TPGS, D: Drug + Pluronic ${ }^{\circledR}$ F-68, E: Drug + Pluronic ${ }^{\circledR}$ F-127 and F: Drug + SLS

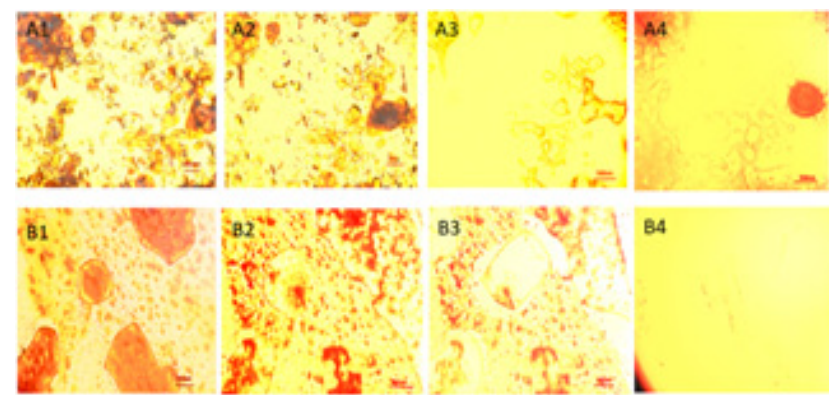

Fig. 2: HSM images of A: SLS and drug at A1: Initial $\left(32{ }^{\circ} \mathrm{C}\right)$, A2: $100{ }^{\circ} \mathrm{C}, \mathrm{A} 3: 200{ }^{\circ} \mathrm{C}, \mathrm{A} 4: 240{ }^{\circ} \mathrm{C}$; B: TPGS and drug at B1: Initial $\left(32{ }^{\circ} \mathrm{C}\right), \mathrm{B} 2: 40{ }^{\circ} \mathrm{C}, \mathrm{B} 3: 100{ }^{\circ} \mathrm{C}, \mathrm{B} 4: 200{ }^{\circ} \mathrm{C}$

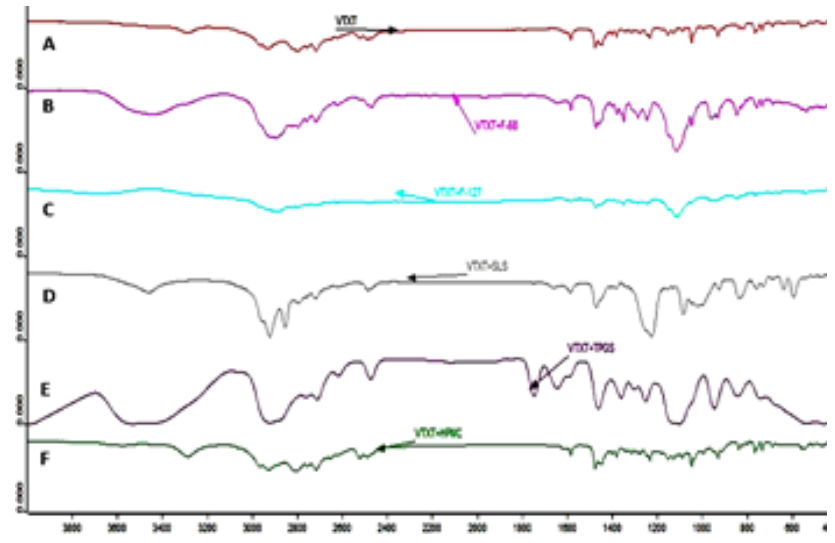

Fig. 3: FTIR spectra of A: Drug, B: Drug + Pluronic ${ }^{\circledR}$ F-68, C: Drug + Pluronic ${ }^{\circledR}$ F-127, D: Drug + SLS, E: Drug + TPGS and F: Drug + HPMC

\subsection{Preparation of nanosuspension}

\subsubsection{Screening of stabilizers}

For screening of stabilizers one variable at a time approach was used in which one process/ formulation factor was changed at a time while keeping all other parameters constant. Nanosuspension formulation was commenced on $600 \mathrm{rpm}$ by screening stabilizers namely Pluronic ${ }^{\circledR}$ F-127, Pluronic ${ }^{\circledR}$ F-68, SLS, HPMC and TPGS. Particle size and polydispersity index (PDI) were main parameters based on which screening was done. Amongst all the stabilizers screened, SLS and Pluronic ${ }^{\circledR}$ F-127 provided smaller size and PDI (Table 2) as compared to others and were taken further. During storage, nanosuspension containing SLS as stabilizer showed lower increase in size as compared to batch containing Pluronic ${ }^{\circledR}$ F-127 at room temperature. Therefore considering changes in particle size of prepared nanosuspension SLS was selected for further study. SLS is an ionic stabilizer which involves the adsorption of ionic charges on the surface of particles resulting in mutual repulsive forces between particles. Anionic nature of SLS provides the higher electrostatic repulsive force as compared to other stabilizers whereas Pluronics are known for steric stabilization provided by them ${ }^{[34]}$.

Table 2: Effect of various processing conditions on size and PDI of drug nanocrystals

\begin{tabular}{|c|c|c|c|}
\hline \multicolumn{2}{|c|}{ Nano-precipitation method } & $\begin{array}{l}\text { Average } \\
\text { particle size } \\
\text { (in nm) }\end{array}$ & PDI \\
\hline \multirow{4}{*}{ Solvent } & DCM & $340.18 \pm 26.1$ & $0.444 \pm 0.21$ \\
\hline & Methanol & $469.1 \pm 41.3$ & $0.552 \pm 0.21$ \\
\hline & Ethanol & $327.0 \pm 21$ & $0.425 \pm 0.42$ \\
\hline & Acetone & $212.2 \pm 22.1$ & $0.123 \pm 0.24$ \\
\hline \multirow{5}{*}{$\begin{array}{c}\text { Drug } \\
\text { concentration }\end{array}$} & $1 \mathrm{mg} / 10 \mathrm{ml}$ & $410.3 \pm 31.4$ & $1.00 \pm 0.23$ \\
\hline & $2 \mathrm{mg} / 10 \mathrm{ml}$ & $339.1 \pm 46.7$ & $0.588 \pm 0.5$ \\
\hline & $3 \mathrm{mg} / 10 \mathrm{ml}$ & $380.9 \pm 17.5$ & $0.512 \pm 0.33$ \\
\hline & $4 \mathrm{mg} / 10 \mathrm{ml}$ & $332.9 \pm 35.2$ & $0.384 \pm 0.57$ \\
\hline & $5 \mathrm{mg} / 10 \mathrm{ml}$ & $203 \pm 11.7$ & $0.184 \pm 4.74$ \\
\hline \multirow{3}{*}{$\begin{array}{l}\text { Solvent to } \\
\text { anti-solvent } \\
\text { ratio }\end{array}$} & $1: 3$ & $145.1 \pm 13$ & $0.428 \pm 0.23$ \\
\hline & $1: 5$ & $215.9 \pm 9.35$ & $0.173 \pm 0.31$ \\
\hline & $1: 10$ & $385.5 \pm 11.8$ & $0.193 \pm 0.24$ \\
\hline \multirow{5}{*}{ Stabilizer } & $\begin{array}{l}\text { Pluronic }^{\circledR} \\
\text { F-68 }\end{array}$ & $415.0 \pm 9.7$ & $0.970 \pm 0.76$ \\
\hline & $\begin{array}{l}\text { Pluronic }^{\circledR} \\
\text { F-127 }\end{array}$ & $203 \pm 11.7$ & $0.184 \pm 0.3$ \\
\hline & SLS & $220.9 \pm 21.3$ & $0.153 \pm 0.16$ \\
\hline & TPGS & $334.6 \pm 31$ & $0.382 \pm 0.29$ \\
\hline & HPMC & $\begin{array}{l}\text { Visible } \\
\text { precipitate }\end{array}$ & - \\
\hline \multirow{4}{*}{$\begin{array}{l}\text { Drug to } \\
\text { stabilizer } \\
\text { ratio }\end{array}$} & $0.5: 1$ & $456.3 \pm 18.1$ & $0.535 \pm 0.62$ \\
\hline & $1: 5$ & $615.2 \pm 31.2$ & $0.518 \pm 0.21$ \\
\hline & $1: 1$ & $237.2 \pm 8.12$ & $0.213 \pm 0.31$ \\
\hline & $1: 3$ & $528.7 \pm 25.6$ & $0.414 \pm 0.17$ \\
\hline
\end{tabular}

\subsubsection{Screening of solvent and solvent to anti-} solvent ratio

The solvent for formulation preparation were screened from methanol, acetone, ethanol, and DCM. Nanosuspension made with methanol showed visible precipitate probably as the drug was least soluble in methanol. Amongst all the solvents tried, acetone provided the size and PDI in acceptable range and hence acetone was selected for further for optimization of solvent: antisolvent ratio. 
Solvent (any organic solvent used to dissolve drug) to anti-solvent (aqueous phase) ratio was optimized by taking three ratios viz. 1:3, 1:5 and 1:10. As the supersaturation is an important phenomenon which depends upon the evaporation and miscibility of solvent in anti-solvent used, it was important to assess this parameter. From all the ratios screened 1:5 ratio gave adequeate size and PDI and was taken further for optimization of other parameters.

\subsubsection{Optimization of drug to stabilizer ratio}

The effect of various concentrations of stabilizer on the particle size and PDI was studied by preparing nanosuspension using $0.5: 1,1: 1,1: 3$ and $1: 5$ ratio of drug to stabilizer respectively. It was found that, the $1: 1$ ratio of drug to stabilizer resulted in nanosuspension with smallest size and PDI. This could be due to the fact that in case of $0.5: 1,1: 3$ and $1: 5$ ratio, the concentration of stabilizer was sufficient to initiate solubilization phenomenon. On the basis of these results 1:1 drug to stabilizer ratio with SLS was chosen as optimized stabilizer level.

\subsubsection{Optimization of stirring speed}

Effect of stirring speed on particle size and PDI of prepared nanosuspension was observed and optimized batches were obtained at $600 \mathrm{rpm}$ speed as other rpm screened $(400,800,1000)$ resulted in visible precipitate. (Figure 4) shows original graph for size distribution by intensity using DLS technique.
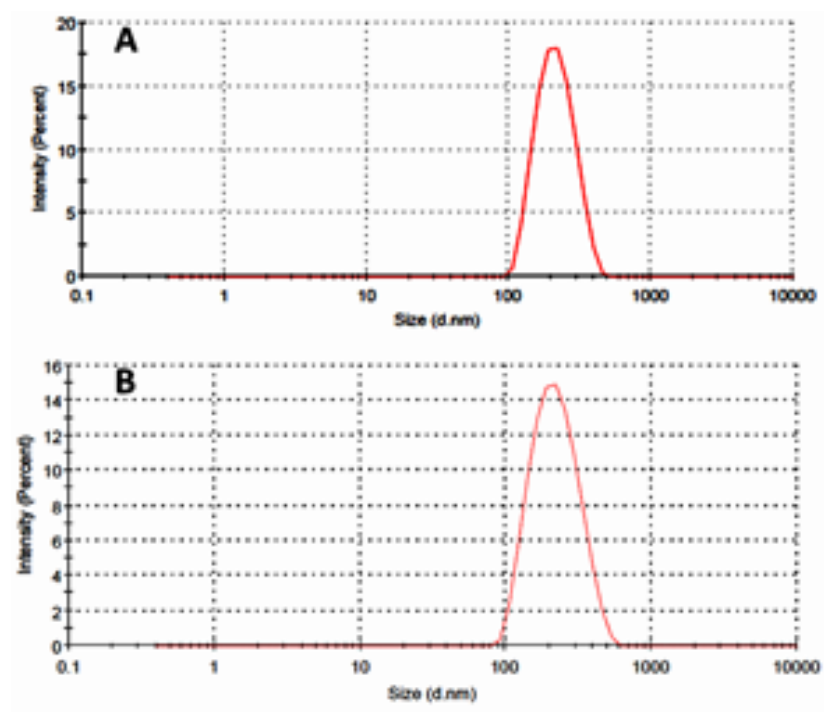

Fig. 4: Size distribution of VOR nanosuspension prepared utilizing optimized parameters with A: SLS and B: Pluronic ${ }^{\circledR}$ F127

\subsubsection{Lyophilization of optimized nanosuspension}

Optimization of type as well as concentration of cryoprotectants often requires studies with large number of formulations. Even though lyophilization process is expensive, freeze-thaw studies are rapid and quicker in comparison to lyophilization and thus screening of type and concentration of cryoprotectants can be carried out easily. According to freeze-thaw study, an effective cryoprotectant is the excipient which protects formulation during the freezing step of lyophilization and the same is determined by calculating $\mathrm{Sf} / \mathrm{Si}$ ratio (where $\mathrm{Sf}$ and $\mathrm{Si}$ are final size and initial size of the formulation respectively) $)^{[23,35]}$.

The cryoprotectants were screened in initial trial in the concentration ranges of $1 \%, 2 \%, 5 \%$, and $10 \%$. After carrying out a freeze-thaw study the ratio of the initial size (Si) to the final size (Sf) was plotted against individual group and the cryoprotectant that gave the smallest ratio was finally selected. Amongst all the cryoprotectants, 1\% trehalose was selected as final cryoprotectant to work with (Figure 5).

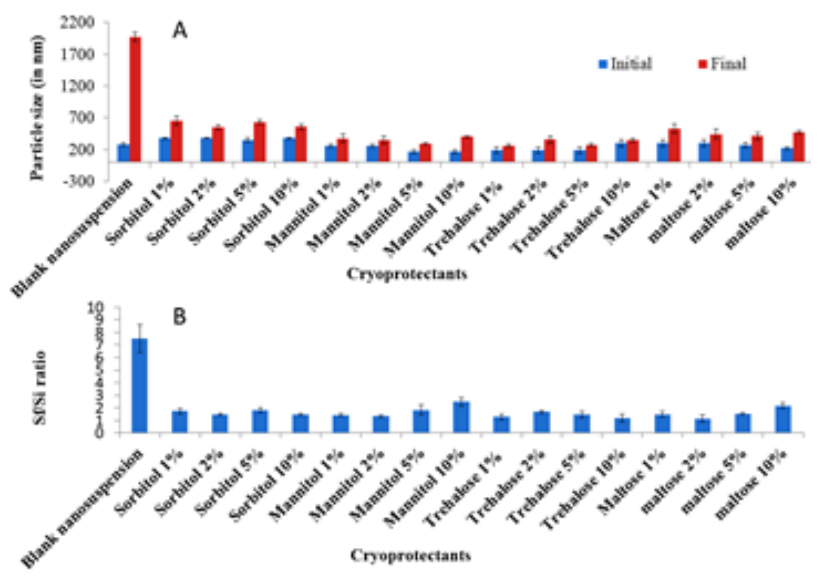

Fig. 5: Screening of cryoprotectants by freeze thaw studies

\subsubsection{Formulation characterization}

\subsubsection{Particle size and zeta potential analysis}

The lyophilized nanocrystals (particularly weighed amount) was re-dispersed in Milli ${ }^{\circledR} \mathrm{Q}$ water and particle size, zeta potential, PDI was determined using DLS method. Average particle size, PDI and zeta potential of finally optimized nanosuspension were found to be $241.6 \pm 7.9 \mathrm{~nm}, 0.163 \pm 0.12$ and $-7.8 .1 \pm 0.53 \mathrm{mV}$ respectively. Both the size as well as PDI were obtained in acceptable range ${ }^{[36]}$. Zeta potential plays an important role in the stability of nanosuspension and is a measure of electrostatic stabilization.

\subsubsection{Particle morphology and surface roughness analysis}

SEM analysis was carried out to study the surface morphology of lyophilized nanocrystals, untreated drug, 
physical mixture, and lyophilized nanocrystals without any cryoprotectant. Irregularities on the surface was found in case of untreated drug, while the lyophilized formulation was found to be smoother in appearance. Morphology obtained in lyophilized nanocrystals was quite different from appearance of physical mixture.

Surface roughness analysis of lyophilized nanocrystals in comparison to unprocessed drug and physical mixture was performed with the help of Atomic Force Microscopy (AFM) AFM analysis results showed that particle size of the untreated drug was more than lyophilized nanocrystals. (Figure 6) presents the AFM images of the untreated drug, physical mixture, lyophilized nanocrystals and lyophilized nanocrystals without cryoprotectants. The results revealed that both the particle size as well as surface roughness of untreated drug was found to be greater than lyophilized nanocrystals.

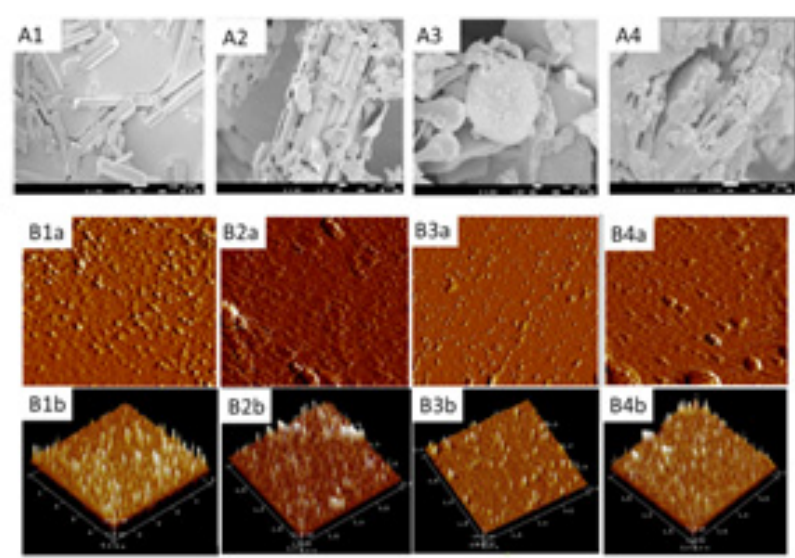

Fig. 6: A: SEM images of A1: Drug, A2: Physical mixture, A3: Lyophilized nanocrystals, A4: Lyophilized nanocrystals without cryoprotectant; B: AFM images of B1: Drug, B2: Physical mixture, B3: Lyophilized nanocrystals and B4: Lyophilized nanocrystals without cryoprotectant

\subsubsection{Physical/chemical state characterization}

DSC and XRD were conducted to assess the physical state of drug present. Results obtained from DSC analysis of lyophilized nanocrystals revealed absence of endothermic peak for drug. Probable reason for that could be either decrease in crystallinity of drug or merging of small endothermic event of drug in thermal event of SLS. Further XRD was conducted to confirm the physical state and results obtained suggested absence/decrease in peak intensity for drug, which again indicates decrease in crystallinity of the drug (Figure 7). Previously authors have reported drug amorphization after freeze dried $\operatorname{processing}^{[37]}$.

FTIR analysis of lyophilized nanocrystals revealed presence of all the peaks of drug which shows no chemical modifications after all the processing (Figure 8).

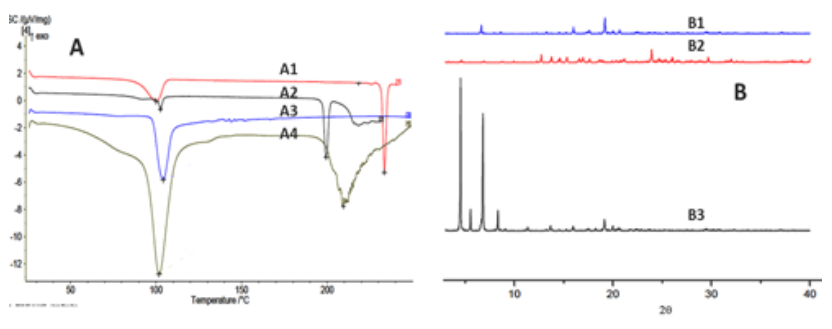

Fig. 7: A: DSC thermograms of A1: Drug, A2: SLS, A3: Trehalose, A4: Lyophilized nanocrystals and B: XRD spectra of B1: Drug, B2: Lyophilized nanocrystals, B3: Physical mixture

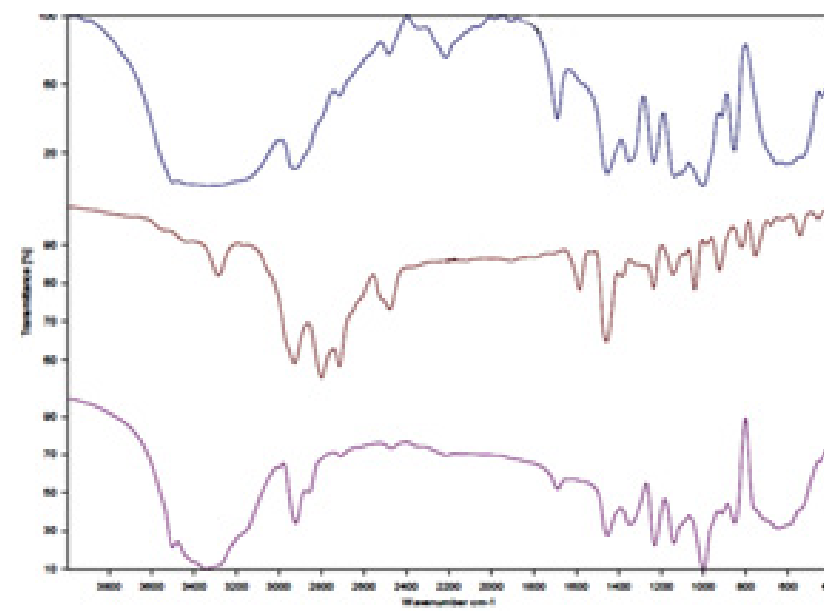

Fig. 8: FTIR spectra of A: Lyophilized nanocrystals, B: Drug and C: Physical mixture

\subsubsection{Analysis of drug content}

The lyophilised sample was analysed for the drug content with validated analytical method described in previous section by adding pre-weighed sample $(1 \mathrm{mg} /$ $\mathrm{ml})$ to solvent. Drug assay was calculated for lyophilized nanocrystals and the value was found to be $98.73 \pm 1.2$ $\%$. Experiment was conducted in triplicate and result is expressed as mean $\pm \mathrm{SD}$.

\subsubsection{Formulation of Orally Disintegrating Tablets (ODTs)}

Evaluation of powder characteristics was carried out and bulk density, tapped density, angle of repose, Hausner's ratio, and compressibility index, were calculated (Table 3). Values obtained for Angle of repose, Hausner's ratio and Carr's index suggested that powder blend possess good flow properties as well as compressibility.

Table 3: Evaluation of powder blend for compressibility and flow properties

\begin{tabular}{cc}
\hline Parameter & Values obtained \\
\hline Bulk density & $0.381 \mathrm{~g} / \mathrm{ml}$ \\
Tapped density & $0.457 \mathrm{~g} / \mathrm{ml}$ \\
Angle of repose & $28^{\circ}$ \\
Hausner's ratio & 1.19 \\
Carr's index & $16 \%$ \\
\hline
\end{tabular}




\subsubsection{Evaluation of ODTs}

Prepared ODTs were evaluated for hardness, wetting time, water absorption ratio, disintegration time and weight variation (Table 4). Parameters tested were found to be in the pharmacopoeial limits. Weight variation was found to be less than $10 \%$ whereas friability values were less than $1 \%$. Hardness was found to be $3.5 \pm 0.27 \mathrm{~kg} / \mathrm{cm}^{2}$ whereas wetting time and water absorption ratio (R) was found to be $10.5 \pm 1.37 \mathrm{sec}$ and $92.16 \pm 15.04 \%$ respectively. Images acquired at different time points during wettability test are depicted in (Figure 9). Disintegration time was in the range of $19.8 \pm 0.15 \mathrm{sec}^{[38,39]}$.

Table 4: Characteristics of prepared tablets

\begin{tabular}{cc}
\hline Parameters & $\begin{array}{c}\text { Tablet prepared with } \\
\text { lyophilized nanocrystals }\end{array}$ \\
\hline Hardness $\left(\mathrm{kg} / \mathrm{cm}^{2}\right)$ & $3.5 \pm 0.27$ \\
Thickness $(\mathrm{mm})$ & $3.5 \pm 0.35$ \\
Weight variation $(\%)$ & $<7.5 \%$ \\
Disintegration time $(\mathrm{sec})$ & $19.8 \pm 0.15$ \\
Friability $(\%)$ & $1 \%$ \\
Wetting time $(\mathrm{sec})$ & $10.5 \pm 1.37$ \\
\hline
\end{tabular}
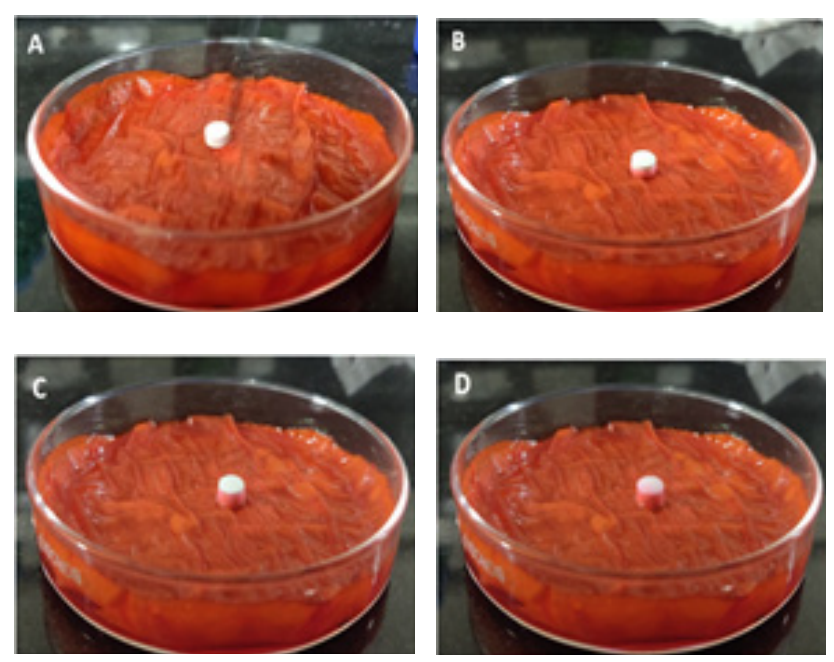

Fig. 9: Images from wettability testing at A: initial, B: $5 \mathrm{sec}, \mathrm{C}$ : $8 \mathrm{sec}$ and D: $11 \mathrm{sec}$ after complete wetting

\subsubsection{In-vitro release study}

In vitro drug release study was carried out for prepared ODT formulations. Along with it ODT compressed using untreated drug was also compared. Results are shown in (Figure 10). Drug release of ODTs formulated using lyophilized nanocrystals was found to be more as compared to ODTs formulated using the untreated drug in SSF due to solubility advantage provided by nanocrystals. Whereas dissolution profile for both these ODTs formulation was found to be, approximately higher in $0.1 \mathrm{~N} \mathrm{HCl}$ increase in SSF medium in comparison with untreated verses lyophilized ODTs was prominent as well. Higher release in $\mathrm{HCl}$ media could be attributed to high solubility of drug at lower $\mathrm{pH}$.
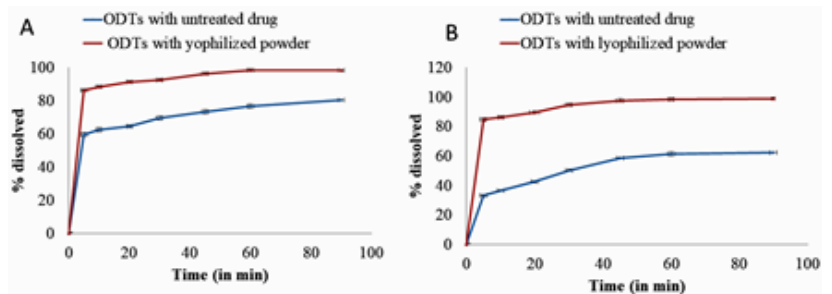

Fig. 10: Dissolution studies of ODTs prepared with untreated drug and Lyophilized nanocrystals in A: $0.1 \mathrm{~N} \mathrm{HCl}$ and B: SSF

\section{CONCLUSION}

Nanosuspension formulation of drug VOR (with average particle size, PDI and zeta potential values of 215.9 $\pm 9.35 \mathrm{~nm}, 0.123 \pm 0.31,-19.3 \pm 2.24 \mathrm{mV}$ respectively) was successfully prepared by nano-precipitation method with SLS as a surfactant. Nanosuspension was lyophilized with $1 \%$ trehalose as cryoprotectant of choice. The lyophilised powder was characterized using DSC, FITR, SEM, AFM and XRD analysis to asess surface morphology, roughness, physical state of drug present and chemical modifications. In vitro release studies revealed that about $\sim 97 \%$ of release of drug for the ODTs prepared with nanocrystals as compared to unprocessed drug $(\sim 60 \%)$ within same time. Thus the overall developed formulation found to have potential for solubility enhancement of drug VOR using nanoprecipitation technique.

\section{ACKNOWLEDGEMENT}

This research work was supported by National Institute of Pharmaceutical Education and Research (NIPER)Ahmedabad under the financial funding of Ministry of Chemicals and Fertilizers, India.

\section{ABBREVIATIONS}

AFM: Atomic force microscopy

BCS: Biopharmaceutical classification system

CMC: Critical micelle concentration

DCM: Dichloromethane

DSC: Differential scanning calorimetry

HPH: High-pressure homogenization

HPMC: Hypromellose

ICH: International conference on harmonization

SSF: Simulated salivary fluid

PDI: Polydispersity index 
MDD: Major depressive disorder

WHO: world health organization

RCS: Refrigerated cooling system

SEM: Scanning electron microscopy

SLS: Sodium lauryl sulfate

TD: Trehalose dihydrate

VOR: Vortioxetine

XRD: X-ray diffraction

\section{CONFLICT OF INTEREST}

There are no conflicts of interest.

\section{REFERENCES}

1. Belmaker, R. and G. Agam, Major depressive disorder. New England Journal of Medicine, 2008. 358(1): p. 55-68.

2. Otte, C., et al., Major depressive disorder. Nature Reviews Disease Primers, 2016. 2: p. 16065.

3. Nettleton, J.A., Omega-3 fatty acids and health. 1995: Springer Science \& Business Media.

4. Kessler, R.C., et al., The epidemiology of major depressive disorder: results from the National Comorbidity Survey Replication (NCS-R). Jama, 2003. 289(23): p. 3095-3105.

5. Geddes, J.R., et al., Relapse prevention with antidepressant drug treatment in depressive disorders: a systematic review. The Lancet, 2003. 361(9358): p. 653-661.

6. D'Agostino, A., C.D. English, and J.A. Rey, Vortioxetine (Brintellix): a new serotonergic antidepressant. Pharmacy and Therapeutics, 2015. 40(1): p. 36.

7. Garnock-Jones, K.P., Vortioxetine: a review of its use in major depressive disorder. CNS drugs, 2014. 28(9): p. $855-874$.

8. Pearce, E.F. and J.A. Murphy, Vortioxetine for the treatment of depression. Annals of Pharmacotherapy, 2014. 48(6): p. 758-765.

9. He, S.-F., et al., An investigation into vortioxetine salts: crystal structure, thermal stability, and solubilization. Journal of pharmaceutical sciences, 2016. 105(7): p. 2123-2128.
10. Bhatt, A. and A. Pethe, Nanotechnology: a promising drug delivery for poorly water soluble drugs. J Pharm Res, 2010. 3: p. 1748-51.

11. Tambe, V., et al., Surface engineered dendrimers in siRNA delivery and gene silencing. Current pharmaceutical design, 2017. 23(20): p. 2952-2975.

12. Thakkar, S., et al., Nanocrystal based drug delivery system: conventional and current scenario. Recent patents on nanotechnology, 2017. 11(2): p. 130-145.

13. Junyaprasert, V.B. and B. Morakul, Nanocrystals for enhancement of oral bioavailability of poorly water-soluble drugs. asian journal of pharmaceutical sciences, 2015. 10(1): p. 13-23.

14. Pund, S. and A. Joshi, Nanoarchitectures for Neglected Tropical Protozoal Diseases: Challenges and State of the Art, in Nano-and Microscale Drug Delivery Systems. 2017, Elsevier. p. 439-480.

15. Sun, B. and Y. Yeo, Nanocrystals for the parenteral delivery of poorly water-soluble drugs. Current Opinion in Solid State and Materials Science, 2012. 16(6): p. 295-301.

16. Komati, S., et al., QbD-based design and characterization of mucoadhesive microspheres of quetiapine fumarate with improved oral bioavailability and brain biodistribution potential. Bulletin of Faculty of Pharmacy, Cairo University, 2018. 56(2): p. 129145.

17. Balestrieri, F., et al., Application of differential scanning calorimetry to the study of drug-excipient compatibility. Thermochimica acta, 1996. 285(2): p. 337-345.

18. Taneja, S., S. Shilpi, and K. Khatri, Formulation and optimization of efavirenz nanosuspensions using the precipitation-ultrasonication technique for solubility enhancement. Artificial cells, nanomedicine, and biotechnology, 2016. 44(3): p. 978-984.

19. Van Eerdenbrugh, B., et al., Alternative matrix formers for nanosuspension solidification: dissolution performance and X-ray microanalysis as an evaluation tool for powder dispersion. European Journal of Pharmaceutical Sciences, 2008. 35(4): p. 344-353.

20. Wang, Y., et al., Stability of nanosuspensions in drug delivery. Journal of controlled release, 2013. 172(3): p. 1126-1141.

21. Saez, A., et al., Freeze-drying of polycaprolactone and poly (D, L-lactic-glycolic) nanoparticles induce 
minor particle size changes affecting the oral pharmacokinetics of loaded drugs. European Journal of Pharmaceutics and Biopharmaceutics, 2000. 50(3): p. 379-387.

22. Dave, V., et al., Formulation design and optimization of novel fast dissolving tablet of chlorpheniramine maleate by using lyophilization techniques. Bulletin of Faculty of Pharmacy, Cairo University, 2017. 55(1): p. 31-39.

23. Gol, D., S. Thakkar, and M. Misra, Nanocrystal-based drug delivery system of risperidone: lyophilization and characterization. Drug development and industrial pharmacy, 2018. 44(9): p. 1458-1466.

24. Shah, R.B., M.A. Tawakkul, and M.A. Khan, Comparative evaluation of flow for pharmaceutical powders and granules. Aaps Pharmscitech, 2008. 9(1): p. $250-258$.

25. Naiem, N.H., E.A. Essa, and G.M. Elmaghraby, ENHANCING DISSOLUTION RATE OF INDOMETHACIN BY IN SITU CRYSTALIZATION; DEVELOPMENT OF ORALLY DISINTEGRATING TABLETS. International Journal of Pharmacy and Pharmaceutical Sciences, 2018. 10(5): p. 18-23.

26. Sharma, D.J.I.p., Formulation development and evaluation of fast disintegrating tablets of salbutamol sulphate for respiratory disorders. 2013. 2013.

27. Kuno, Y., et al., Effect of the type of lubricant on the characteristics of orally disintegrating tablets manufactured using the phase transition of sugar alcohol. 2008. 69(3): p. 986-992.

28. Prajapati, B.G. and B.J.I.J.P.T.R. Patel, Formulation, evaluation and optimization of orally disintegrating tablet of piroxicam. 2010. 2(3): p. 1893-9.

29. Chen, Y., et al., Formulation and evaluation of a montelukast sodium orally disintegrating tablet with a similar dissolution profile as the marketed product. 2017. 22(2): p. 168-172.
30. Pabari, R. and Z.J.J.o.y.p. Ramtoola, Effect of a disintegration mechanism on wetting, water absorption, and disintegration time of orodispersible tablets. 2012. 4(3): p. 157-163.

31. Basu, B., et al., Formulation and evaluation of fast dissolving tablets of cinnarizine using superdisintegrant blends and subliming material. 2011. 2(4): p. 266.

32. Anup, N., S. Thakkar, and M. Misra, Formulation of olanzapine nanosuspension based orally disintegrating tablets (ODT); comparative evaluation of lyophilization and electrospraying process as solidification techniques. Advanced Powder Technology, 2018. 29(8): p. 1913-1924.

33. Yamashita, T. and K. Takatsuka, Hydrogen-bond assisted enormous broadening of infrared spectra of phenol-water cationic cluster: An ab initio mixed quantum-classical study. The Journal of chemical physics, 2007. 126(7): p. 074304.

34. Shete, G., et al., Stabilizers used in nano-crystal based drug delivery systems. Journal of Excipients and Food Chemicals, 2016. 5(4).

35. Date, P.V., A. Samad, and P.V. Devarajan, Freeze thaw: a simple approach for prediction of optimal cryoprotectant for freeze drying. AAPS PharmSciTech, 2010. 11(1): p. 304-313.

36. Lieberman, S., M.G. Enig, and H.G. Preuss, A review of monolaurin and lauric acid: natural virucidal and bactericidal agents. Alternative \& Complementary Therapies, 2006. 12(6): p. 310-314.

37. Einfalt, T., O. Planinšek, and K. Hrovat, Methods of amorphization and investigation of the amorphous state. Acta Pharmaceutica, 2013. 63(3): p. 305-334.

38. US Pharmacopoeia. 2014, United States Pharmacopoeial Convention. p. 342-351, 1571-72.

39. Indian Pharmacopoeia. 1996, Government of India, Ministry of Health \& Family Welfare, Controller of Publication. p. 2: A144-A145. 